\title{
La ley de correspondencia
}

\begin{abstract}
T $\mathrm{N}$ marzo de 19.76, miembros de la comunidad académica del Instituto Tecnológico Autónomo de México me distinguieron con una invitación para presentar mi libro recientemente publicado Desarrollo, crisis e ideología en la formación del capitalismo. Basado en algunas de las ideas que expuse en esa ocasión y que me pareció despertaron mayor interés, escribo este breve ensayo.
\end{abstract}

Uno de los temas más importantes que se manejan en las ciencias sociales es la posible existencia de leyes que gobiernen los procesos históricos. Mucho se ha escrito sobre este tema sin que se hayan producido avances satisfactorios. Reflexionando sobre el problema, considero que una de esas posibles leyes es la Ley de Correspondencia y a explorar ésta se dedicó la investigación que dio lugar a este libro. Esta ley tiene una gran importancia en todas las ciencias sociales pero especialmente en la Economía, pues hay una tendencia entre los especialistas que se dedican a esta disciplina a considerar que existen procesos económicos químicamente puros y no contaminados por factores extraeconómicos.

Las sociedades humanas funcionan simultáneamente en varios niveles, tales como el ecónomico, el político o el ideológico y la Ley de Correspondencia postula que las transformaciones que se operen en cualquiera de estos niveles provocan cambios en los otros. Dicho en otra forma, una sociedad es un conjunto de un gran número de elementos que no están simplemente yuxtapuestos sino que están vinculados y que por tanto los cambios que experimente uno de esos elementos se comunican a los demás modificando el conjunto. 
A partir de esta afirmación inicial se pueden hacer una serie de derivaciones de gran interés. Cada uno de los niveles de la actividad social del hombre forma un todo en el cual los diferentes elementos que lo integran sólo son inteligibles en relación al conjunto. En el nivel económico esto a menudo se soslaya y se examinan elementos aislados como si tuvieran un funcionamiento independiente desvinculado de cualquiera de los otros. Así, existen estudios, por ejemplo, del funcionamiento de los mecanismos financieros sin referencia a los sistemas productivos a los cuales están superpuestos. Estos análisis presentan una porción de la realidad que por minoritaria ha perdido gran parte de su significado. Pero la estructura económica es, a su vez, únicamente una parte de un conjunto mayor, una totalidad que es la única que goza de verdadera inteligibilidad ya que integra todos los agentes cuya actuación determina el comportamiento de las diversas partes incluyendo los mecanismos financieros.

Esto nos conduce a preguntarnos acerca de cuántos y cuáles serían esos niveles del funcionamiento social del hombre. Sobre este particular las respuestas pueden diferir de un autor a otro y dichas diferencias pueden ser legítimas. En el modelo de la Ley de Correspondencia que presentamos, la totalidad está integrada por seis niveles diversos, cada uno de los cuales es un conjunto de elementos vinculados entre sí de manera que podemos calificarlo de estructura. Algunos de estos conjuntos ya reciben en el lenguaje ordinario el nombre de estructuras y así se habla de estructura económica y estructura social, pero todos los seis niveles constituyen otras tantas estructuras y es necesario considerar, por ejemplo, la ideología como tal aun cuando no se acostumbre describirla de esa manera. Los seis niveles que integran el modelo son: la ecosis, la estructura económica, la estructura social, el poder político y la ideología en sus dos niveles: el consciente y el inconsciente.

La ecosis es una categoría que expresa la interacción entre las comunidades humanas y el entorno natural que habitan. Esta relación es recíproca. Es obvio que el medio natural con su orografia, su clima, acceso al mar, etcétera, influye decisivamente sobre la sociedad que se asienta en él. Menos evidente, pero de importancia no menor, es la acción de la comunidad sobre el medio. Incluso los pueblos primitivos dotados de técnicas poco avanzadas pueden causar cambios importantes a través de procesos de desforestación, ensalinamiento, construcción de canales, etcétera, y desde luego la influencia de sociedades equi- 
padas con la tecnología contemporánea es mucho mayor. Esta relación puede ser positiva o negativa. En el primer caso hay un creciente dominio del hombre sobre el medio que no se traduce en un deterioro de éste, mientras que en el segundo, o bien hay una pérdida del control social sobre su entorno o un deterioro de éste que a un plazo más o menos largo terminará por minar dicho control. Tenemos pues una ecosis positiva y una negativa. Por otra parte, los progresos de la Ecología en las últimas décadas no dejan duda sobre el carácter estructural del medio natural y las relaciones humanas con él. Una y otra vez se ha demostrado cómo alteraciones en un factor, tal como la introducción de plaguicidas en la agricultura, tienen repercusiones en el conjunto, muchas veces inesperadas por no haberse previsto los incontables y sutiles nexos entre los componentes que configuran dicho medio.

El funcionamiento de la estructura económica es probablemente el más estudiado ya que, como es bien sabido, ha sido objeto de un análisis sistemático desde la aparición en la segunda mitad del siglo XVIII de la Escuela Clásica de Economía. Pese a este gran esfuerzo no puede asegurarse que haya sido mejor comprendida que los otros niveles como lo comprueban las continuas controversias que rdividen a los especialistas. Lo más importante que puede afirmarse es la ya mencionada interdependencia entre los distintos elementos que configuran la economía de una sociedad.

La estructura social también ha sido examinada sistemáticamente y muchas de las categorias que utilizan los especialistas, tales como clases sociales, movilidad social, etcétera, han pasado a enriquecer el lenguaje cotidiano. El uso de métodos matemáticos en el análisis ha contribuído a minimizar el papel de la subjetividad y darle un mayor rigor.

El estudio del poder político ha logrado avances importantes tanto en el examen de su principal manifestación, el Estado, como en el de otras instancias que como las iglesias, los partidos políticos y los grupos de presión, configuran también una estructura aun cuando este término no se utilice generalmente para designarlo.

Por último, tenemos dos niveles cuyo estudio presenta dificultades de un orden diferente. En este modelo el término ideología tiene un significado muy amplio ya que se refiere a la totalidad de las creenciàs y sentimientos que predominan en una sociedad haciendo abstracción de su contenido de verdad, ya 
que ésta, como se muestra a través del libro, experimenta una evolución que nos lleva a afirmar su carácter eminentemente histórico. Las ideas que albergan las mentes humanas tienen características muy interesantes desde la perspectiva de nuestro análisis. En primer lugar, dichas ideas también forman un conjunto estructural en el cual los elementos no están aislados sino que existe una tendencia a formar un conjunto congruente que los vincula entre sí. Así, por ejemplo, una sociedad en que se tiene respeto por la ciencia y su método tenderá a aceptar las ideas nuevas cuando éstas sean acordes con los principios científicos y rechazará o por lo menos devaluará las que no sean congruentes con dichos principios. Lo mismo ocurrirá en el caso de una comunidad sminada por una idea religiosa, etcétera. Se puede objetar que a menudo tanto una persona como una sociedad tienen ideas contradictorias. Esto es cierto y nos conduce a llevar el análisis más lejos siguiendo dos directrices.

Por un lado, hay que decir que las contradicciones se presentan en el seno de todos los niveles. La afirmación de que los elementos que integran cada nive stán estructurados no debe interpretarse en el sentido de negar las contradicciones entre ellos, sino simplemente como la negación de la independencia de los mismos. Los exámenes de la realidad convencen rápidamente a cualquier observador de que las contradicciones. no sólo existen sino que proliferan en el interior de cada estructura. En efecto, el consumo a menudo abusivo de los recursos no renovables es un ejemplo de las contradicciones que se dan en el nivel de la ecosis y que a menudo ha conducido a la pérdida del control de una comunidad sobre el entorno natural que habita, como trágicamente ha registrado varias veces la Historia, desde los ensalitrados campos de la antigua Mesopotamia hasta el Tazón de Polvo (Dust Bowl) del oeste estadounidense. El examen de los otros niveles demuestra lo mismo. La continua necesidad, a menudo angustiosa, que se presenta de elegir entre la inversión y el consumo nos muestra una de las principales contradicciones en el nivel económico. Los diferentes grupos y clases que integran la estructura social mantienen continuas luchas por ocupar posiciones de privilegio que implican contradicciones más o menos irreductibles. Por otra parte, tal vez la mayor contradicción social sea la que surge entre el individuo y el anhelo que siente de libertad y la necesidad que experimenta la sociedad de limitar ésta en persecución del bien común. Cosa similar ocurre con el poder político en donde las fuerzas de la democracia y el 
autoritarismo libran combates continuos. Podemos concluir esta serie de afirmaciones declarando que la Ley de Correspondencia no es el mecanismo a través del cual se genere un desarrollo armónico de la sociedad, sino, por el contrario, la expresión más acabada de las innumerables contradicciones que permean toda su evolución.

Por otra parte, es preciso aclarar que en las sociedades existen ideas que integran una ideología dominante y otras minoritarias y sumergidas, que son patrimonio de sectores sociales más o menos amplios o incluso de individuos aislados. Es indudable que para los propósitos de nuestro modelo, la atención debe centrarse en la ideología dominante. Esto se entiende mejor si tenemos presente que las ideas no son únicamente elementos determinados sino que también lo son determinantes. En efecto, las sociedades lo mismo que los individuos actúan de acuerdo con las ideas que consideran correctas y, por tanto, una sociedad tenderá a comportarse de acuerdo con la ideológía dominante, esto es, la integrada por ideas, verdaderas o falsas, que tengan una posición hegemónica.

Por último, tenemos el nivel del inconsciente. En el libro que presentamos, éste no está analizado separadamente pues se le presentó formando parte de un nivel ideológico que compendía tanto las ideas que forman el patrimonio de la conciencia como aquellos impulsos que integran el dominio del inconsciente. Hacia fines del siglo XIX y principios del XX varios avances científicos establecieron una brecha insalvable entre la ciencia decimonónica y la de nuestra época. Como ejemplo se podrían citar los casos de la Física Cuántica y la Teoría de la Relatividad que pusieron en crisis el paradigma del universo que había quedado establecido por las leyes enunciadas por Isaac Newton. En igual nivel se ubica el descubrimiento del inconsciente por Sigmund Freud y sus seguidores, que derrumbó el axioma subyacente desde la antigüedad en el estudio del hombre que considera éste como un ser exclusivamente racional. Emergió a la luz del conocimiento la existencia, hasta entonces ignorada, de un complejo conjunto de dinámicas sumergidas más allá de las fronteras de la conciencia y la racionalidad que determinan la conducta humana en una contienda secreta con éstas.

Los grandes iniciadores de la Revolución Sicoanalítica, Sigmund Freud y Carl Gustav Jung, se refirieron a la existencia de un inconsciente individual que integra la personali- 
dad de cada ser humano, pero también plantearon que existe un inconsciente colectivo que opera sobre comunidades y masas humanas. El estudio del inconsciente individual fue recibido como una valiosa herencia por la Medicina y otras disciplinas e impulsado hasta convertirse en un elemento central de la Siquiatría y la Sicología actuales, pero el tema del inconsciente colectivo no recibió una bienvenida semejante de parte de sus destinatarios naturales, los científicos sociales, economistas, politólogos, historiadores, etcétera, con lo que se originó lo que nos parece grave pérdida para dichas ciencias. Consideramos que el inconsciente colectivo integrado por el conjunto de mecanismos síquicos sumergidos más allá de la conciencia de una sociedad y compartidos por un número de sus integrantes lo suficientemente grande para convertirse en un factor para su actuación colectiva es otro nivel que debe considerarse para integrar la totalidad que es el verdadero objeto del conocimiento de todas las ciencias sociales. Es el sexto nivel del modelo que asociamos a la Ley de Correspondencia.

Al contemplar los seis niveles que componen la totalidad social debe evitarse la idea de que éstos constituyen seres individuales que simplemente mantienen vínculos entre sí a través de los cuales se ejercen influencias de un nivel a otro de manera que un cambio en uno de ellos se refleja en los demás. Esta es una imagen inadecuada de la realidad que no hace justicia al carácter totalizador de ésta. Ciertamente muchos elementos parecen pertenecer tan sólo a un nivel y esto corresponde a la visión que hemos señalado, la cual individualiza los niveles, pero en muchos otros casos hay elementos que existen simultáneamente en varios niveles o incluso en todos ellos. Un ejemplo clarificará esto. Un ferrocarril es un elemento económico y su aparición potencia la capacidad productiva de un territorio, pero simultáneamente ese mismo ferrocarril es un factor de control político que permitirá quẹ un gobierno central ejerza por primera vez un dominio efectivo de esa región que antes escapaba a su control. Es obvio que dicho ferrocarril es al mismo tiempo un elemento económico y un elemento político. Por otra parte, el ferrocarril en cuestión ejercerá una infuencia determinante en otros niveles, pero no es fácil afirmar que es un elemento de ellos. Así, la disponibilidad de ese medio de transporte puede conducir a la explotación de bosques hasta la desforestación sin ser por ello un elemento de la ecosis sino tan sólo un determinante de la misma; igualmente puede favorecer 
un proceso de organización que altere en favor de la población urbana el antiguo equilibrio social, de esta manera el ferrocarril es un factor que determina la estructura social pero no es un componente de la misma; otro tanto puede decirse de los niveles ideológicos donde muchas ideas o sentimientos vinculados con la antigua vida agraria seguramente cambiarán al intensificarse el urbanismo y los contactos con el exterior.

Lo anterior ayuda a explicar la inevitabilidad del cambio global al ocurrir una transformación en algún nivel y al mismo tiempo los rezagos y resistencias que se generan en otros. Si un elemento pertenece simultáneamente a varios niveles es obvio que éstos se modificarán inmediatamente al introducirse ese factor novedoso en una sociedad dada, pero otros niveles a los cuales no pertenece en sí y para los cuales únicamente es un determinante, pueden oponer y de hecho oponen una resistencia al cambio que origina rezagos y tensiones que crecerán al incrementarse el retraso. Esto nos hace enfatizar dos características de gran importancia en el funcionamiento de la Ley de Correspondencia. Por una parte, está su carácter objetivo que la convierte en la expresión de una inevitabilidad independiente de la voluntad humana y, por otra parte, el hecho fundamental, al cual ya nos hemos referido, de que esta ley no implica en forma alguna un desarrollo armónico de la sociedad en el que cada cambio es inmediatamente acompañado por otros que le devuelven el equilibrio, sino, por el contrario, la expresión de innumerables contradicciones que forman parte de la evolución social y de los conflictos que generan llenando con su registro las páginas de la Historia.

Por último, debemos referirnos a un punto importante. El uso adecuado de la Ley de Correspondencia proporciona una gran fuerza explicativa a las diversas ciencias sociales, lo cual parece ser el principal beneficio que se deriva de ella. Sin embargo, no ocurre otro tanto con la capacidad predictiva de las mismas cuyo incremento debido al uso de esta ley no es significativo. Los agentes del cambio son demasiado numerosos y sus relaciones excesivamente complejas para permitir escapar a la incertidumbre que parece ser la principal característica de los procesos sociales. El análisis de este tema donde se conjugan las eternas interrogantes sobre el determinismo y la libertad, sigue mereciendo la atención de los diversos especialistas de las ciencias que estudian la sociedad y sus transformaciones. 